\title{
XXXVIII. A method of producing an intense cadmium spectrum, with a proposal for the use of mercury and cadmium as standards in refractometry
}

\section{T. Martin Lowry D.Sc. F.C.S.}

To cite this article: T. Martin Lowry D.Sc. F.C.S. (1909) XXXVIII. A method of producing an intense cadmium spectrum, with a proposal for the use of mercury and cadmium as standards in refractometry , Philosophical Magazine Series 6, 18:104, 320-327

To link to this article: http://dx.doi.org/10.1080/14786440808636704

\section{9.}

\section{Submit your article to this journal $\longleftarrow$}

山 Article views: 2

$\mathbf{Q}^{\mathbf{2}}$

View related articles 
XXXVIII. A Method of producing an intense Cadmium Spectrum, with a proposal for the use of Mercury and Cadnium as Standards in Refractometry. By T. Martin Lowry, D.Sc., F.C.S.*

$\mathrm{O}^{\mathrm{F}}$

$F$ the different line spectra that are available for spectroscopic standards-hydrogen, mercury, cadmium, \&c.the simplest and purest is undoubtedly the cadmium spectrum. The visible spectrum is made up of four strong lines (red, green, blue, and dark blue), which are so narrow and of such a high degree of purity in respect of the absence of satellites that they have been used by Michelson to produce interferencebands of an order of retardation that bas apparently never been reached in the case of any other lines. Michelson's measurements of the wave-lengths of the three chief Cadmium lines :-

$$
\begin{array}{llcc}
\text { Cd red ........ } & 6438 \cdot 4722 & 10^{-10} \text { metre. } \\
\text { Cd green ..... } & 5085 \cdot 8240 & , & , \\
\text { Cd blue ..... } & 4799 \cdot 9107 & , & ,
\end{array}
$$

have indeed formed the standards from which all other wavelengths have been deduced. It is therefore evident that the cadmium spectrum is destined to play an extremely important part in optical determinations of all kinds. Unfortunately, the difficulty of producing a cadmium lamp which shall burn steadily and give out light of high intensity has been so great that the four cadmium lines have been used only very occasionally in optical experiments.

\section{Sodium.}

The standard monochromatic light employed aimost universally for refractometric and polarimetric measurements has been the yellow flame-spectrum of sodium, which has the advantage of being produced with very great readiness, but with all the drawbacks inseparable from the use of a doublet, instead of a single line, as a standard. Thus in determining the refractive index $n_{\mathrm{D}}$ of a liquid, the Pulfrich refractometer gives readings for the less refrangible constituent, whilst a hollow prism mounted on a spectroscope gives an average value for the two constituents, unless indeed the resolution be sufficient to read them separately. In polarimetric work the double character of the sodium line render it impossible to secure a proper extinction for large values of $\alpha_{D}$, since one

* Communicated by the Physical Society : read June 25, 1909. 
Method of producing an intense Cadmium Spectrum. 321

wave-length is transmitted with considerable intensity when the other is extinguished, and in addition there is always some uncertainty as to the "optical mass-centre" of the doublet, which may indeed vary in different types of sodium-lamp, on account of changes in the relative intensity of the two constituents *. It should also be noted that the sodium flame emits a considerable amount of light of other colours, which in accurate work, or in reading large rotations, must be removed by filtering through a coloured screen, or, better, by means of a spectroscopic eyepiece (Perkin).

\section{Hydrogen.}

The hydrogen lines,

$$
\begin{aligned}
& \mathrm{H}_{\alpha} \text { (red), w.-l. }=6560 \cdot 04, \\
& \mathrm{H}_{\beta} \text { (blue), w.-l. }=4861 \cdot 49, \\
& \mathrm{H}_{\gamma} \text { (violet), w.-l. }=4340 \cdot 66,
\end{aligned}
$$

have been universally employed with the sodium doublet in refractometric work when dispersion-values were required. The choice has been wholly one of convenience and has no other merit to recommend it. The vacuum-tube, though easily fitted up, can hardly be seriously considered as a source of light. The red line is by far the strongest, and has been used with advantage to produce interference-fringes in measurements of length $\dagger$, but would be utterly useless for polarimetric work in which the source of light must be viewed through a Nicol's prism set within $2^{\circ}$ or $3^{\circ}$ of the extinction position. The violet line is unpleasantly weak even for refractometric measurements, and demands the use of the full power of a six-inch coil, with an efficient optical condenser, before readings can be made with any degree of comfort. The hydrogen spectrum has the further disadvantage of showing, at least in an ordinary vacuum-tube, an almost continuous back-ground of weak lines. Although, therefore, refractometers are regularly sent out with tables for the sodium and the three hydrogen lines-and no data whatever for light of any other wave-length-it is evident that this position is radically unsound and cannot be permanently maintained.

* Compare Landolt, Optische Drehungsvermögen, 1898, pp. 362 et seq.

+ See, for instance, Tutton's measurements of the coefficients of expansion and of elasticity of crystal-plates (Phil. Trans. 1903, A. 202, p. 143). Compare also Tutton, Proc. K. S., June 10, 1909. 


\section{Choice of Standards.}

The essential properties for a standard source of light are, (1) that it should be of sufficient intensity to be used for all the various types of optical measurements, so that, for instance, refractive indices and optical and magnetic rotatory powers may be determined for the same wave-lengths, (2) that it should be strictly monochromatic and as far as possible free from satellites, and (3) that it should be produced with sufficient readiness to render it generally available. These requirements, as has been shown, are only partially fulfilled by sodium light and fail completely in the case of the hydrogen spectrum. The purpose of the present communication is to suggest that the spectra of mercury and of cadmium fulfil most of the essential conditions outlined above, and to describe a method by which the cadmium spectrum may be rendered more generally available for spectroscopic work.

The suggestion-which is made on the basis of practical experience in the actual measurement of optical and magnetic rotations and of refractive indices for a large range of wavelengths (see for instance Proc. Roy. Soc. 1908, 81. p. 472) - that sodium should give place to mercury and cadmium as a chief standard source of light, is fully supported by the theoretical considerations recently advanced by Bates ("Spectrum Lines as Light Sources in Polariscopic Measurements," Bureau of Standards, Bulletin, 1906, ii. p. 239) and by Nutting ("Polarimetric Sensibility and Accuracy," ibid. p. 249, "Purity and intensity of Monochromatic Light Sources," ilid. p. 439). The former author has worked out a formula showing the errors due to the use of a doublet in polarimetry, and has redetermined the ratio of the sodiumyellow and mercury-green rotations for quartz; the latter has developed formulæ in reference to polarimetric sensibility, and spectral purity. The two points in these papers that bear directly on the practical problem now under consideration are, (1) the confirmation by Bates of the purity of the mercury green line, which gave very sharp readings in the case of a quartz plate of about $5 \frac{1}{2} \mathrm{~mm}$. thickness; this point is, however, seriously discounted by the fact that he professes to read the sodium doublet to $0.0001^{\circ}$, and gives the ratio of sodium to mercury to six significant figures $(0.850944: 1),(2)$ the statement by Nutting that on one basis of reckoning the "spectral purities" of cadmium green, mercury green, and sodium yellow, are represented by the ratios $\frac{1}{100,000}, \frac{1}{10,000}$, and $\frac{1}{700}$, whilst on another basis the "specific 
impurities" of the mercury green and sodium yellow lines are given by the ratios $\frac{1}{1,000,000}$, $\frac{1}{2000}$; these figures serve to show that the change of principal standard now proposed on the basis of practical polarimetric work is fully justified by minute spectroscopic tests on the lines thernselves.

\section{Mercury.}

The use of the enclosed mercury arc as a source of light in spectroscopy dates back to 1860 (J. H. Gladstone, "On the Electric Light of Mercury," Phil. Mag. [4] xx. pp. 249-253), but its use in polarimetric measurements was apparently introduced by Disch (Ann. Phys. (4) xii. p. 1155) in 1903, who made use of the Arons lamp. The Bastian mercury lamp, which has been in use in my own laboratory since 1906, and at the Central Technical College since 1907, has the advantage of being a commercial article of much lower cost; it is constructed with a suitable resistance in the holder, so that it can be plugged into the ordinary lighting circuit without using a resistance-frame or any special leads. This lamp is unfortunately no longer on the market, though it is still constructed to order by the Brush Electrical Engineering Company; but silica lamps of moderate price are promised which may prove to be as economical in working as, and even more efficient in illumination than, the earlier glass lamps.

Of the six chief mercury lines,

$$
\begin{aligned}
& \left.\begin{array}{l}
5790 \cdot 49 \\
5769 \cdot 45
\end{array}\right\} \text { a yellow doublet, } \\
& \left.\begin{array}{l}
5460 \cdot 97 \\
4358 \cdot 58 \text { a splendid green line, } \\
4078 \cdot 03 \\
4046 \cdot 78
\end{array}\right\} \text { at the extreme limit of the visible spectrum, }
\end{aligned}
$$

two, the green and the violet, have already proved to be of the utmost value in polarimetry, and are likely in the future to prove of equal value in the measurement of refraction and dispersion.

Their use in polarimetry has been due to the following considerations. For accurate measurements of the specific rotatory power of a substance, and to any even larger extent for tracing the course of chemical changes (isomeric change, sugar-hydrolysis, \&c.) by polarimetric observations, it is essential to use an intense source of light in association with a very small half-shadow angle, since only thus can a maximum of sensitiveness be secured : it is also desirable to use a light 
of relatively short wave-length, in order that the actual readings may be large *, without incurring the loss of optical intensity and the fatigue which result from the use of blue light. The intense green mercury line, which can be read with a considerably smaller half-shadow angle, and gives readings about 15 per cent. larger than the sodium doublet, is therefore much superior to the traditional standard, apart altogether from the question of spectral purity. In the latter respect the contrast is extreme ; in the case of quartz I have been able, without any noticeable loss of accuracy, to secure readings showing a total rotation of 50 right angles for the mercury green line, two independent series of determinations giving average values $4487.78^{\circ}$ and $4487.79^{\circ}$; sodium under similar conditions gave no extinction at all. The green mercury line promises, indeed, wholly to replace the sodium doublet as a chief standard in polarimetric work, and it is highly desirable that it should acquire as quickly as possible a like predominance in the measurement of refractive indices, and in all other optical determinations.

The violet mercury line has proved indispensable in the measurement of rotatory dispersion on account of its extraordinary brilliancy. In spite of the low sensitiveness of the eye for light of such small wave-length it has been found possible to read this line with a half-shadow angle of only $6^{\circ}$, and to secure series of readings (each an average of 10 settings) which only differed from one another by a hundredth of a degree. The violet line is less pure than the green, as it is accompanied by two satellites of smaller wave-length, but these are so weak that they cannot be seen at all in the polarimeter, and cannot, therefore, produce any appreciable disturbance in the readings. The yellow doublet is made up of two lines separated by about three times as great an interval as in the case of sodium; for small rotations they may be read as one line, but I have also been able, by using a narrow slit, to read them separately; they are, however, altogether unsuited for general use.

For refractometer work the mercury lines are at least as easily available as those of hydrogen; a warmed vacuumtube containing a drop of mercury gives the lines with greater brilliance than those of hydrogen, and it is therefore not unreasonable to suggest that-as a minimum concession to the correlation of optical measurements of various kindsthe use of $\mathrm{H}_{\beta} 4861$ and $\mathrm{H}_{\gamma} 4341$ shall be abandoned in favour of $\mathrm{Hg} 5461$ and $\mathrm{Hg} 4359$ in future refractometric work, and

* As a rule the specific rotation is doubled on passing from yellow to violet. 
that tables for these wave-lengths shall be supplied as a matter of course with instruments of the Pulfrich pattern. It may be noted that the mercury and hydrogen violet lines differ by only 18 Angström units, the nercury line having the longer wave-length: in a Pulfrich instrument the two lines are indistinguishable, but the edge that is read with a hydrogen-mercury vacuum-tube (such as is sometimes sent out with the instrument) is due to mercury and not hydrogen. The adaptation of a polarimeter for use with mercury light costs about $£ 2$, with a further $£ 3$ for the lamp.

\section{Cadmium.}

The cadmium spectrum is much less easy to produce than that of mercury. Michelson made use of a strongly heated vacuum-tube with aluminium electrodes connected to platinum wires passing through the glass. This was improved upon by Hamy (Comptes Rendus, 1897, cxxiv. p. 749) who used a copper heating-jacket and external electrodes, thus avoiding the risk of cracking the hot glass by wires passing through it. I have had no personal experience of such lamps, but am doubtful whether they would give a sufficiently intense light for use in polarimetry. The amalgam lamp with an arc enclosed in silica can be made to give a splendid series of lines for use in spectroscopy, but I have found that it is useless for polarimetric work, since even the green cadmiumline can only be read with a half-shadow angle of nearly $20^{\circ}$. Apparently the current is carried mainly by the mercury, and the other metals show only weakly in the spectrum. I have not been able as yet to find any description of an enclosed cadmium arc, though I believe they have been tried -evidently not with complete success, or the results would be more widely known.

The method set out below is not put forward as the ideal way of producing an.intense cadmium spectrum, but rather as an intermediate stage in the development of the perfect cadmium lamp of the future. It was found that brilliant spectral lines could be sent into the polarimeter by using an arc burning between metallic poles rotating in opposite directions. Copper, for instance, gave a valuable series of lines, and brass electrodes were found to be very efficient for developing a zinc spectrum, the red line $\mathrm{Zn} 6364$, and the

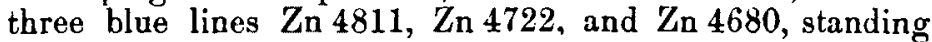
out very distinctly from the copper lines. A brilliant cadmium spectrum could be produced by melting the metal onto copper electrodes, but it soon burned off, and in any case it

Phil. Mag. S. 6. Vol. 18. No. 104. Aug. 1909. Z Z 
was difficult to avoid a displacement of the readings by the appearance of the copper line $\mathrm{Cn} 5106^{\circ}$, as a ghostly partner of the green cadmium line Cd 5086. After many unsuccessful attempts a workable method of producing the cadmium spectrum was found in the use of an alloy of silver and cadmium. It is perhaps not very widely known that these metals are isomorphous, and form an excellent series of alloys. These have the advantage that no eutectic is formed, the melting-points throughout the series lying above that of cadmium and over a considerable range approximating somewhat closely to the melting-point of silver. Thus whilst the addition of 28 per cent. of copper ( $\left.\mathrm{mp} .1082^{\circ}\right)$ lowers the melting-point of silver from $960^{\circ}$ to $780^{\circ}$, the addition of 28 per cent. of cadmium (mp. $322^{\circ}$ ) only lowers the meltingpoint to $860^{\circ}$. Nearly 50 per cent. of cadmium must be added to lower the melting-point to $780^{\circ}$, and even a 60 per cent. alloy melts as high as $700^{\circ}$. These alloys, which can be turned up like pure silver, were supplied by Messrs. Johnson and Matthey in the form of rods $\frac{1}{4}$ inch in diameter and $1 \frac{1}{4}$ inch long.

For spectroscopic work a tiny arc can be burnt quite steadily between the points of the rods, in great contrast to the behaviour of pure cadmium, which splutters very badly and gets choked up with oxide, even when the current is kept so small as not to melt the metal.

For polarimetric work a greater intensity of light is desirable, and this is obtained by using a heavier current, and rotating the electrodes in opposite directions (compare Baly, Spectroscopy, p. 370) in order to maintain the arc in a central position. The rods of alloy were screwed for half their length into copper cylinders 5 inch in diameter, which served the double purpose of cooling the electrodes-a point of some importance-and connecting them with the iron spindles by means of which the rotation was produced. When run at the highest intensity both rods become red hot, and one of the copper cylinders is usually luminous, but it is not desirable to over-run the are since even if the electrodes do not melt the cadmium distils out irregularly and causes a certain amount of spluttering.

The electrodes are filed up before the arc is started, and are carefully adjusted so as to run true to centre; alternatively they may be allowed to burn until the ends are flat and then used without further attention except to adjust the length of the arc from time to time. The cadmium spectrum thus produced is of great brilliance-the green line is even brighter than that of mercury, and can be read with a halfshadow angle of $3^{\circ}$ or less. From some points of view 
it would be a better chief standard than mercury green, as it is considerably brighter and gives readings about 15 per cent. higher, but in view of the greater trouble involved in producing the light, and persuading it to burn steadily, it is better to use it as a secondary line for the study of rotatory dispersion. The red and blue lines are also very bright and can be read quite easily. The dark blue line is of much less intensity, and is not likely to be widely used, as it is difficult to read, and does not differ sufficiently in wave-length from the light blue line to justify the extra trouble involved ; this observation applies, however, only to the existing arrangements, as it is quite possible that when a more powerful source of steady light is available the dark blue line may prove to be of considerable value in shortening the gap between $\mathrm{Cd} 4800$ and $\mathrm{Hg} 4359$.

Unlike copper the silver spectrum does not clash at all with that of cadmium; the brilliant silver green lines

$$
\begin{aligned}
& \begin{array}{l}
5471 \cdot 72 \\
5465 \cdot 66 \\
5209 \cdot 25
\end{array} \\
& \text { doublet (compare sodium) }
\end{aligned}
$$

are separated from the cadmium green, Cd 5086 , by an interval nearly as great as that which separates the two cadmium blues, and the only other line that shows at all strongly in the spectrum is a line in the far-violet, perhaps $\mathrm{Ag} 4055$.

In conclusion : It is suggested that the mercury line $\mathrm{Hg} 5461$ should be used as chief standard in optical work of all kinds, and that dispersion should be measured from this line to $\mathrm{Hg}_{4} 4359$ instead of from $\mathrm{H}_{\alpha} 6561$ or $\mathrm{H}_{\beta} 4861$ to $\mathrm{H}_{\gamma} 4341$. As secondary standards are suggested the flame spectra Li 6708 and $\mathrm{Na} 5893$, purified spectroscopically, together with the three cadmium lines $\mathrm{Cd} 6438$, Cd 5086, Cd 4800, giving a well-distributed series of seven wavelengths.

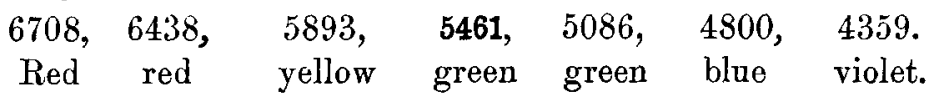

130 Horseferry Road, Westminster, S.W.

Note added July 1909.-I am glad to find that the desirability of a change in the standard wave-lengths for use in refractometry is apparently recognized by other workers : in particular, Dorn \& Lohmann in their measurements of the refractive indices of liquid crystals ( $A \mathrm{nn}$. Physit, June 10th, 1909, [4] xxix. pp. 535-565) have used the series $\mathrm{Li} 6708, \mathrm{Na} 5893, \mathrm{Hg} \mathrm{5461,} \mathrm{Hg} 4359$, which is identical with a series that I am using for the measurement of the refractive dispersions of the alcohols and acids of the aliphatic series, and differs from the series of seven lines used in the measurement of rotatory dispersion only in the omission of the three cadminm lines. 